\title{
Tunable Terahertz Absorption with Optical Tamm State in the Graphene-Bragg Reflector Configuration
}

\author{
Shuai Wang $\mathbb{D}^{1},{ }^{1}$ Guimei Li $\mathbb{D}^{2},{ }^{2}$ and Yanhong Zou $\mathbb{D}^{1}$ \\ ${ }^{1}$ School of Physics and Electronics, Hunan University, Changsha 410082, China \\ ${ }^{2}$ Key Laboratory of Hunan Province for New Retail Virtual Reality Technology, \\ School of Computer and Information Engineering, Hunan University of Commerce, Changsha 410205, China \\ Correspondence should be addressed to Guimei Li; 1507@hnuc.edu.cn and Yanhong Zou; yanhongzou@hnu.edu.cn
}

Received 6 April 2018; Accepted 25 July 2018; Published 5 August 2018

Academic Editor: Qinghua Guo

Copyright (c) 2018 Shuai Wang et al. This is an open access article distributed under the Creative Commons Attribution License, which permits unrestricted use, distribution, and reproduction in any medium, provided the original work is properly cited.

\begin{abstract}
Tunable terahertz absorption in the interface between graphene and dielectric Bragg reflector (DBR) has been numerically demonstrated. The near perfect absorption mainly originates from the enhancement of the electric field owing to the excitation of the optical Tamm state (OTS) at the interface between graphene and dielectric Bragg mirror. It has been found that the absorption peak occurs at specific incident angles, which can be employed for realizing the frequency and angular absorbers. Further, we demonstrate that the position of the absorption peak can be tuned by changing the Fermi energy of graphene. Moreover, the behaviors of the near perfect absorption are strongly related to the dielectric constants and thicknesses of the surrounding dielectrics. The tunability of graphene-DBR structure absorption may help to find favorable applications for the realization of highperformance graphene optoelectronic devices.
\end{abstract}

\section{Introduction}

The electromagnetic absorber is a kind of functional device that can reduce the reflection and transmission of incident light. Recently, terahertz $(\mathrm{THz})$ absorbers have become a hotspot in the field of terahertz $[1,2]$. THz absorbers have a wide range of applications in the field of nanostructures, such as $\mathrm{THz}$ switches [3], thermal detectors [4], time reversal lasers [5], and metamaterial absorbers [6]. To achieve impedance matching we can adjust the structural parameters. And by the mean, the perfect absorption of the incident wave can be obtained. What is more, based on the principle of perfect $\mathrm{THz}$ absorber, we can manufacture electromagnetic switches [7], modulators [8], and sensors [9]. Therefore, many $\mathrm{THz}$ wave absorbers have been studied in recent years. To specify, Tao et al. designed and produced the narrow band absorber of the first $\mathrm{THz}$ band [10]. Since then, $\mathrm{THz}$ double band absorbers, multiband absorbers, and broadband wave absorbers have also been studied [11-13]. However, it is still urgent to design wonderful angular absorber with thin thickness, adjustable angular spectrum for the $\mathrm{THz}$ absorption, and large absorptivity [14].
Graphene has a considerable amount of special properties that attracts scientists' attention, such as high specific surface areas, excellent mechanical properties, remarkable thermal conductivity, and flexibility [15-18]. At the moment, graphene also has excellent optical properties like linear optical properties, nonlinear optical effects, high electrical conductivity, and absorbance interaction [19-24]. With its unique properties being discovered, it has many applications in the field of optical bistability [25], light emission devices [26], and mode-locked fiber lasers [27]. Besides, the graphene has potential applications in $\mathrm{THz}$ absorber as well, such as realizing the tunable $\mathrm{THz}$ absorber based on graphene [28], demonstrating the tunable THz absorption in graphenebased heterostructures [29], and realizing the near perfect absorption by graphene-based modified Otto configuration in the $\mathrm{THz}$ frequency range [30]. More importantly, the conductivity of graphene can be tuned by adjusting gate voltages or chemical doping $[31,32]$, which means that we can obtain different absorption peaks by changing the Fermi energy.

The optical Tamm state (OTS) is a novel interface mode which widely exists at the interface of the photonic crystal 


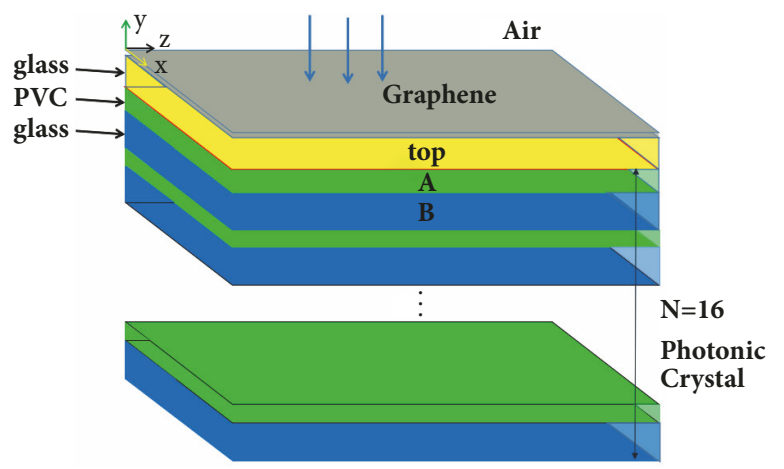

FIGURE 1: The structure diagram of the graphene-DBR structure. The top layer is between graphene and the photonic crystals. A stands for dielectric layer PVC, and B stands for dielectric layer in the photonic crystals with a period number $N=16$, respectively. Here, surface of the graphene layer is defined as the plane of $\mathrm{x}, \mathrm{y}, \mathrm{z}=0$.

(PC) heterostructures [33, 34]. The electric field energy is mainly concentrated on the interface of the dielectric, providing the conditions for almost total absorption of electromagnetic waves. Therefore, in this paper, we propose realizing near perfect absorption of electromagnetic waves by exciting OTS through graphene-DBR structure. Owing to the excitation of OTS and the method of transfer matrix, the structure can achieve almost total absorption in the $\mathrm{THz}$ band. Furthermore, we can achieve near perfect absorption at different wavelengths by adjusting the Fermi energy of graphene, the thickness, and permittivity of the top layer. We believe that the tunable electromagnetic terahertz absorber has potential applications in thermal sensing, THz imaging, and emissivity spectrum modifiers.

\section{Theoretical Model and Method}

We consider that a graphene-DBR structure consists of monolayer graphene, the dielectric photonic crystals (PC) with alternately stacked polyvinyl chloride (PVC) (A) and glass (B), and the top layer glass (with refractive index $n_{\mathrm{s}}=$ 2.3 and thickness $d_{\mathrm{s}}=32 \mu \mathrm{m}$ ) between the monolayer graphene and the photonic crystals as shown in Figure 1. The cycle of PC that we choose is made of 16 pairs of alternate dielectric layer $\mathrm{A}$ (the refractive index $n_{\mathrm{a}}=1.8$ and thickness $d_{a}=42 \mu \mathrm{m}$ ) and dielectric layer $\mathrm{B}$ (the refractive index $n_{b}=2.3$ and thickness $d_{b}=32 \mu \mathrm{m}$ ), and the thickness of graphene is $0.34 \mathrm{~nm}$. It is well known that the conductivity $\sigma$ is responsible for the optical behavior of graphene in the $\mathrm{THz}$ spectral range. The conductivity of graphene can be theoretically described as the Kubo formula Drude-like conductivity [35], namely,

$$
\sigma=\frac{i e^{2} E_{F}}{\pi \hbar^{2}(\omega+i / \tau)}
$$

where $E_{F}$ is the Fermi energy of graphene, $\tau$ is the relaxation time, $\omega$ is the frequency. $e$ and $\hbar$ are the universal constants related to the electron charge and reduced Planck's constant, respectively.
In order to determine the $\mathrm{THz}$ absorption effect, we use the transfer matrix to calculate the reflectance and transmittance, respectively [36]. The $\mathrm{THz}$ waves are propagating across the interface of the two dielectrics. The wave assumes to be $\mathrm{p}$ and $\mathrm{s}$ polarization in the propagated direction. We consider the $\mathrm{p}$ and $\mathrm{s}$ polarization, separately. For the $\mathrm{p}$ polarized, the transmission matrix on adjacent dielectric surfaces can be expressed as

$$
D_{12}=\frac{1}{2}\left[\begin{array}{cc}
1+\eta_{\mathrm{p}}+\varepsilon_{\mathrm{p}} & 1-\eta_{\mathrm{p}}-\varepsilon_{\mathrm{p}} \\
1-\eta_{\mathrm{p}}+\varepsilon_{\mathrm{p}} & 1+\eta_{\mathrm{p}}-\varepsilon_{\mathrm{p}}
\end{array}\right],
$$

where $\varepsilon_{p}=\sigma k_{2 z} / \varepsilon_{0} \varepsilon_{2} \omega, \eta_{p}=\varepsilon_{1} k_{2 z} / \varepsilon_{2} k_{1 z}$, where $\varepsilon_{0}$ is the permittivity in the vacuum and $\varepsilon_{1}$ and $\varepsilon_{2}$ are the dielectric constants, respectively. Similarly, by applying the Ohm's law and boundary conditions, the transfer matrix for s polarized can be expressed as

$$
D_{12}=\frac{1}{2}\left[\begin{array}{cc}
1+\eta_{\mathrm{s}}+\varepsilon_{\mathrm{s}} & 1-\eta_{\mathrm{s}}+\varepsilon_{\mathrm{s}} \\
1-\eta_{\mathrm{s}}-\varepsilon_{\mathrm{s}} & 1+\eta_{\mathrm{s}}-\varepsilon_{\mathrm{s}}
\end{array}\right],
$$

where $\eta_{\mathrm{s}}=k_{2 z} / k_{1 z}, \varepsilon_{\mathrm{s}}=\sigma \mu_{0} \omega / k_{1 z}$, and $\mu_{0}$ is the permeability in the vacuum. According to the transfer matrix, the absorption rate of terahertz wave can be expressed as $\mathrm{A}=1-\mathrm{R}-\mathrm{T}$, where $\mathrm{R}$ and $\mathrm{T}$ are the reflectance and the transmittance, respectively.

\section{Results and Discussions}

In this section, we discuss the characteristics of reflectance and absorption in the graphene-DBR structure. For the sake of simplicity, we only focus on the case of $\mathrm{p}$ polarization and simulate the absorption efficiency of incident electromagnetic wave at the central wavelength $\lambda_{0}=300 \mu \mathrm{m}$ on the structure. As shown in Figure 2, we can see that the wave absorber achieves almost $100 \%$ absorption in the wavelength of $314 \mu \mathrm{m}$ with the Fermi energy $\left(E_{F}\right)$ of $0.85 \mathrm{eV}$. From Figure 2(a), we can see that the wavelength of the absorber changes with the Fermi energy ranging from $0.45 \mathrm{eV}$ to $1.05 \mathrm{eV}$. The dip of graphene-DBR structure's reflectance decreases and then increases as Fermi energy rises. When 


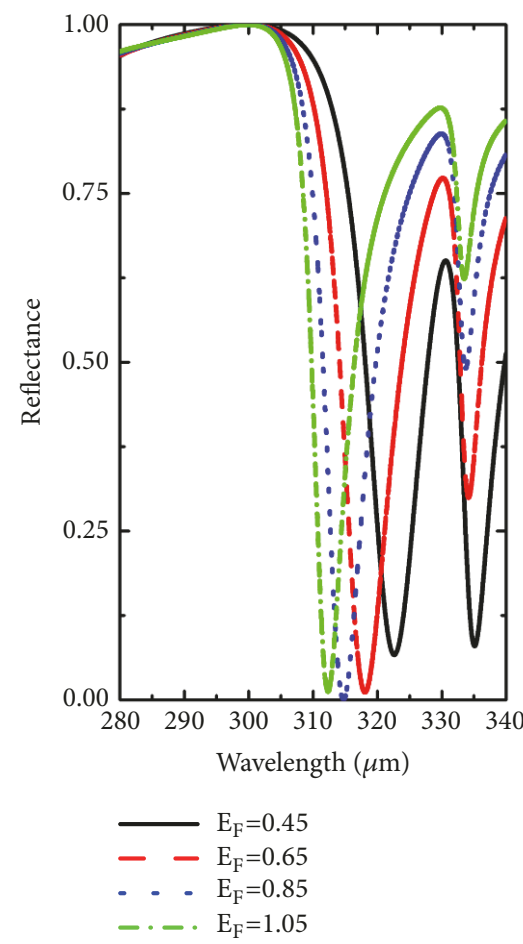

(a)

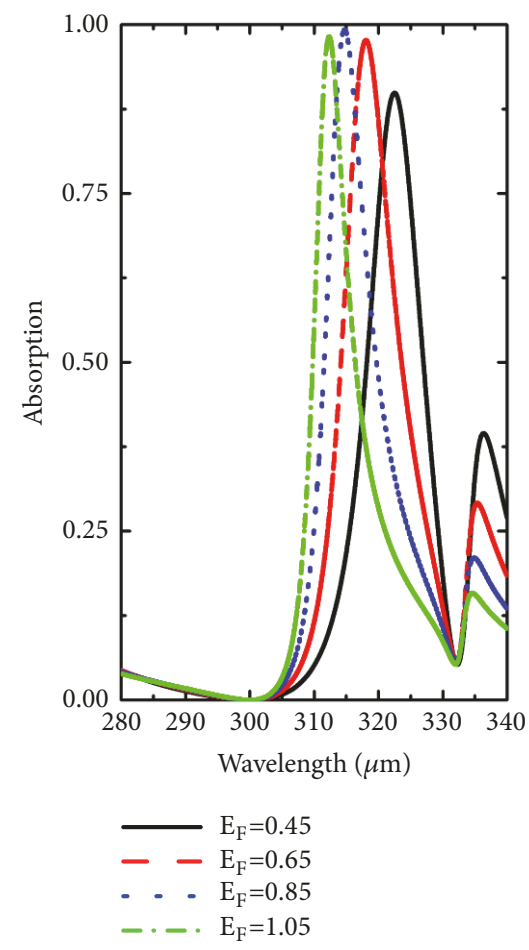

(b)

FIgURE 2: Reflectance and absorption of the graphene-DBR structure as a function of the Fermi energy. (a) Reflectance rate varies with different graphene Fermi energy, where the incident wavelength $\lambda_{0}=300 \mu \mathrm{m}$, and the Fermi energy ranging from $0.45 \mathrm{eV}$ to $1.05 \mathrm{eV}$. (b) Absorption rate varies with different graphene Fermi energy, and the parameters are the same as those in (a).

$E_{F}=0.45 \mathrm{eV}$, the wavelength of the reflectance dip is 322 $\mu \mathrm{m}$. However, for $E_{F}=0.85 \mathrm{eV}$, the wavelength of the reflectance dip is $314 \mu \mathrm{m}$, and the reflectance is almost zero at the minima. But when the Fermi energy increases, the reflectance increases as well. It can be clearly seen that the absorber approaches nearly $100 \%$ absorption in $E_{F}=0.85 \mathrm{eV}$ as shown in Figure 2(b). It is well known that the Fermi energy has an impact on the conductivity of graphene. The imaginary part of the optical conductivity $\operatorname{Im}(\sigma)$ exhibits some complex behaviors: it has a dip near the wavelength $\lambda_{\text {dip }}$ for $\hbar \omega=$ $2 E_{F}$, and at this dip, $\operatorname{Im}(\sigma)$ can become negative. Moreover, dip shifts to shorter wavelength with the increasing Fermi energy $E_{F}$. Compared with traditional metal materials, the biggest advantage of graphene is that its conductivity can be tuned dynamically. The change in Fermi energy of graphene can affect the conductivity of graphene, which affects the absorption of terahertz wave. Therefore, we can adjust the absorption effect of the structure by changing the Fermi energy.

To further understanding the absorption mechanism of OTS, we present the change rule of phase varying with the wavelength, as shown in Figure 3. It is found that the phase $(\varphi)$ is almost zero at $\lambda=314 \mu \mathrm{m}$ due to the reason that the incident electromagnetic wave excited OTS on the structure. The near perfect absorption wavelength is roughly the same as the intrinsic wavelength of OTS. The intrinsic wavelength of OTS can also be deduced according to the theory of transfer matrix. In particular, when the light is confined to the interface between graphene and DBR structure, the OTS is excited. The amplitude matching condition should satisfy $r_{\mathrm{Gra}} r_{D B R} e^{2 i \varphi} \approx 1$, where $r_{\mathrm{Gra}}$ and $r_{D B R}$ are the graphene-top layer interface and top layer-PC interface amplitude reflection coefficient of propagating wave, respectively, and $\varphi$ is the phase change of the propagating wave between the interfaces. As shown in Figure 3, the formula $\operatorname{Arg}\left(r_{G r a} r_{D B R} e^{2 i \varphi}\right) \approx 0$ at the wavelength of $314 \mu \mathrm{m}$. Thus, we can estimate that the wavelength of $314 \mu \mathrm{m}$ equals the intrinsic wavelength of OTS.

In order to better describe how OTS is excited by graphene, Figure 4(a) shows that the electric field along the $\mathrm{z}$ axis in the graphene-DBR structure. By contrast, it also shows the distribution of electric field in the structure without the graphene, as shown in Figure 4(b). The electric field is normalized, and we set the position of graphene as $Z=0$. To simplify the problem, we do not take dielectric loss into consideration; it is found that the electric field is evanescent attenuation for $Z>0$. In addition, it occurs the maximum field in the interface between the air and the graphene. The maximum field strength at $Z=0$ is about 36 times larger than the electric field intensity in Figure 4(b). OTS at the graphene-DBR interface is strongly excited, which is a new surface wave pattern. The monolayer graphene's real part is not enough to change the profile of the field distribution in the structure. But its imaginary part will effectively change the intensity of the electric field; thus, the graphene with thin layer thickness can strongly absorb the incident light as a dissipative layer. Because the OTS is excited in the graphene 


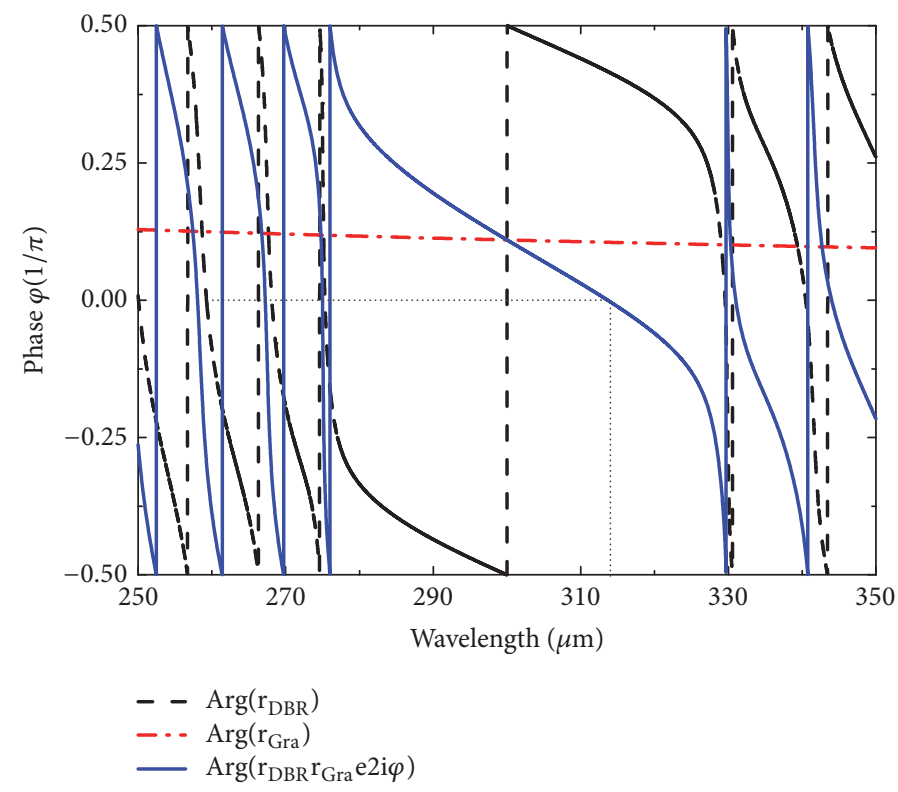

FIGURE 3: The phase of reflection coefficient $r_{G r a}$ (red line) for a graphene-top layer interface, reflection coefficient $r_{D B R}$ (black line) for top layer-PC interface, and $r_{D B R} r_{G r a} e^{2 i \varphi}$ (blue line) with the changing of wavelength.

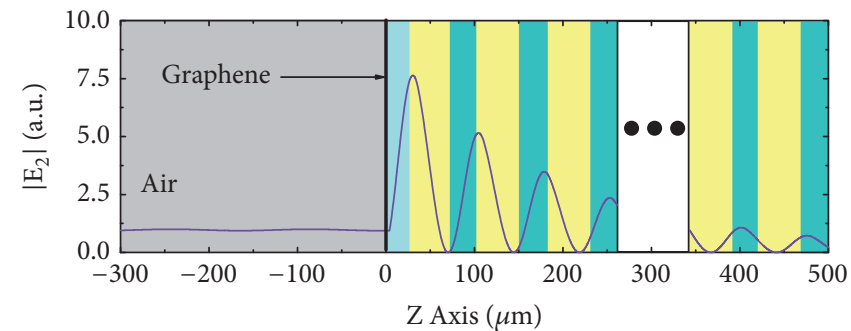

— with graphene

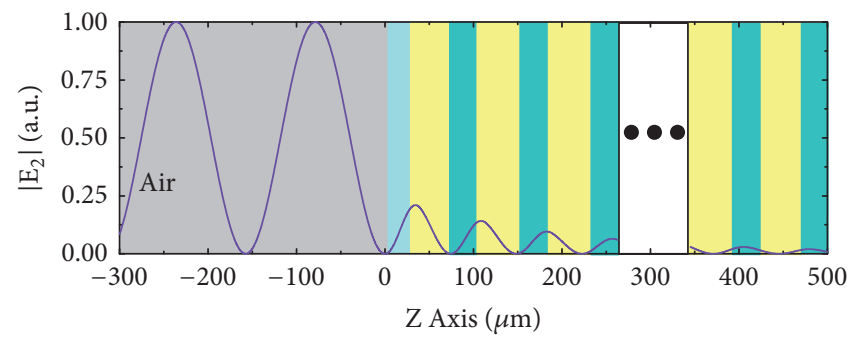

— without graphene

(a)

(b)

FIGURE 4: The normalized electromagnetic field distribution of OTS. (a) The electromagnetic field distribution in the multilayer structure with the covering of graphene. (b) The electromagnetic field distribution in the multilayer structure without the covering of graphene.

layer, the reflectance is almost zero at the wavelength $\lambda=$ $314 \mu \mathrm{m}$.

Aside from the influence of graphene Fermi energy absorption properties, we also discuss the influence of the dispersion characteristics of top layer on graphene-DBR structure absorption. Figure 5(a) shows the change rule of the dielectric constant of top layer for different wavelength absorption. We found that the absorption peak moves in the direction in which the wavelength becomes larger (redshift, corresponding to an increase in wavelength) as the dielectric constant increases. However, the peak absorption efficiency does not change. In addition, Figure 5(b) shows the effect of the thickness of top layer on the absorption efficiency. Kaliteevski et al. have been proposed theoretically and experimentally that the intrinsic energy of OTS varies with the thickness of the top layer [37]. When the thickness of top layer $d_{s} \leq \lambda_{0} /\left(2 n_{s}\right)$, we can only see the presence of an OTS. However, when the thickness of the top layer increases, multiple OTSs will appear in the photonic bandgap. In Figure 5(b), it can obviously observe that, as the thickness rises, the absorption peak moves toward the direction of the larger wavelength. Based on the simulation results above, it is hardly affecting absorption peak at the location of the low frequency, although the absorption peak amplitude can be controlled. On the contrary, the absorption peak has a clear red shift only at the location of the higher frequency. All the absorption efficiency is basically the same. Compared to the dielectric constant, the influence of thickness on the absorption wavelength is relatively larger. However, the absorption bandwidth basically remained constant. We can utilize different parameters to design the wavelength absorbers in the terahertz range. It is also found that the two parameters have their own characteristics on the overall absorption amplitude and absorption peak position; it has potential applications in optical sensors, optical filters, optical switches, and low-threshold optical bistability and even electromagnetically induced transparency. 


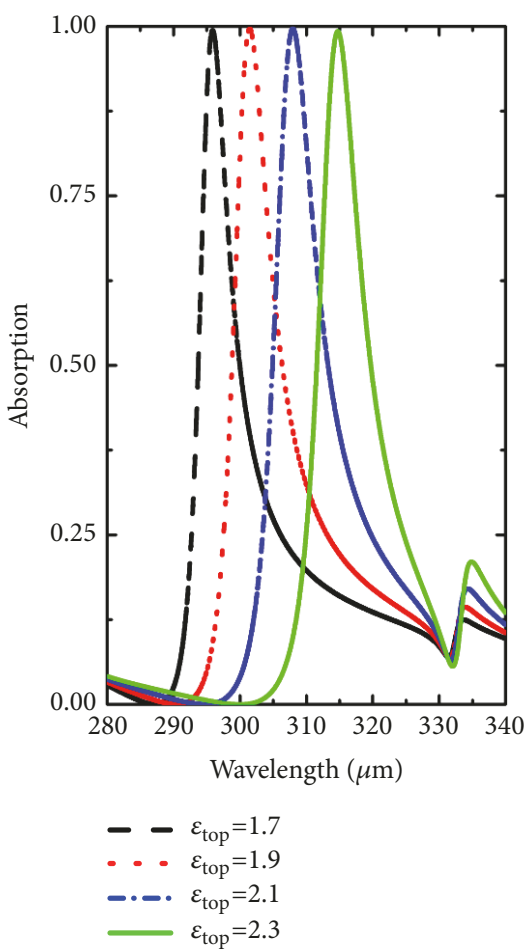

(a)

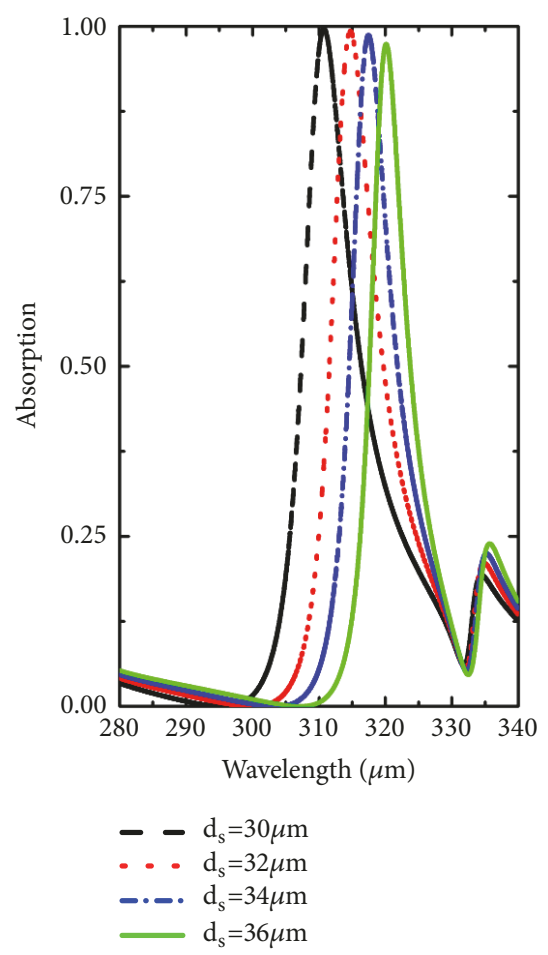

(b)

Figure 5: Reflectance of the graphene-DBR structure as a function of the dielectric constant and thickness. (a) Absorption rate varies with different top layer different dielectric constants, where the dielectric constant ranges from 1.7 to 2.3. (b) Absorption rate varies with different top layer different thicknesses, where the thickness ranges from $30 \mu \mathrm{m}$ to $36 \mu \mathrm{m}$.

\section{Conclusions}

In conclusion, we designed the graphene-DBR structure to achieve the tunable properties of the near perfect absorption in the $\mathrm{THz}$ frequency regime. We found that the different wavelength absorption effect can be altered by adjusting the dielectric constant, thickness of the top layer, or the Fermi energy of graphene. On the basis of the analysis above, near perfect absorption can be achieved at different frequency bands in the structure. These results may contribute to finding promising applications in the $\mathrm{THz}$ frequency, including optical logic devices, optimized narrow band tunable absorbers, optical filters, and optical switches.

\section{Data Availability}

The data used to support the findings of this study are available from the corresponding author upon request.

\section{Conflicts of Interest}

The authors declare that they have no conflicts of interest.

\section{Acknowledgments}

This work is partially supported by the National Nature Science Foundation of China (Grant no. 61378002) and the
Key Laboratory of Hunan Province for New Retail Virtual Reality Technology (2017TP1026).

\section{References}

[1] C. Zhao, H. Zhang, X. Qi et al., "Ultra-short pulse generation by a topological insulator based saturable absorber," Applied Physics Letters, vol. 101, no. 21, Article ID 211106, 2012.

[2] Q. Bao, H. Zhang, Y. Wang et al., "Atomic-layer graphene as a saturable absorber for ultrafast pulsed lasers," Advanced Functional Materials, vol. 19, no. 19, pp. 3077-3083, 2009.

[3] F. Meng, K. Ma, K. S. Yeo, and S. Xu, "Monolithic Sub-Terahertz SPDT Switches With Low Insertion Loss and Enhanced Isolation," IEEE Transactions on Terahertz Science and Technology, vol. 8, no. 2, pp. 192-200, 2018.

[4] X. Cai, A. B. Sushkov, R. J. Suess et al., "Sensitive roomtemperature terahertz detection via the photothermoelectric effect in graphene," Nature Nanotechnology, vol. 9, no. 10, pp. 814-819, 2014.

[5] A. Boh Ruffin, J. Van Rudd, J. Decker et al., "Time reversal terahertz imaging," IEEE Journal of Quantum Electronics, vol. 38, no. 8, pp. 1110-1119, 2002.

[6] N. I. Landy, S. Sajuyigbe, J. J. Mock, D. R. Smith, and W. J. Padilla, "Perfect metamaterial absorber," Physical Review Letters, vol. 100, no. 20, Article ID 207402, 2008.

[7] J. Son, "Terahertz electromagnetic interactions with biological matter and their applications," Journal of Applied Physics, vol. 105, no. 10, p. 102033, 2009. 
[8] M. Liu, X. Yin, E. Ulin-Avila et al., "A graphene-based broadband optical modulator," Nature, vol. 474, no. 7349, pp. 64-67, 2011.

[9] F. Miyamaru, S. Hayashi, C. Otani et al., "Terahertz surfacewave resonant sensor with a metal hole array," Optics Letters, vol. 31, no. 8, pp. 1118-1120, 2006.

[10] H. Tao, N. I. Landy, C. M. Bingham, X. Zhang, R. D. Averitt, and W. J. Padilla, "A metamaterial absorber for the terahertz regime: design, fabrication and characterization," Optics Express, vol. 16, no. 10, pp. 7181-7188, 2008.

[11] Y. Wen, W. Ma, J. Bailey, G. Matmon, X. Yu, and G. Aeppli, "Polarization-independent dual-band terahertz metamaterial absorbers based on gold/parylene-C/silicide structure," Applied Optics, vol. 52, no. 19, pp. 4536-4540, 2013.

[12] Y. Ma, H. Zhang, Y. Li, Y. Wang, W. Lai, and J. Li, "Dual-band and polarization-insensitive terahertz absorber based on fractal Koch curves," Chinese Physics B, vol. 23, no. 5, pp. 594-599, 2014.

[13] X. Shen, Y. Yang, Y. Zang et al., "Triple-band terahertz metamaterial absorber: Design, experiment, and physical interpretation," Applied Physics Letters, vol. 101, no. 15, Article ID 154102, 2012.

[14] C. Liu, J. Ye, and Y. Zhang, “Thermally tunable THz filter made of semiconductors," Optics Communications, vol. 283, no. 6, pp. 865-868, 2010.

[15] Q. Bao and K. P. Loh, "Graphene photonics, plasmonics, and broadband optoelectronic devices," ACS Nano, vol. 6, pp. 36773694, 2012.

[16] H. Zhang, Q. Bao, D. Tang, L. Zhao, and K. Loh, "Large energy soliton erbium-doped fiber laser with a graphene-polymer composite mode locker," Applied Physics Letters, vol. 95, no. 14, Article ID 141103, 2009.

[17] F. Bonaccorso, Z. Sun, T. Hasan, and A. C. Ferrari, "Graphene photonics and optoelectronics," Nature Photonics, vol. 4, no. 9, pp. 611-622, 2010.

[18] Z. Chen, C. Xu, C. Ma, W. Ren, and H.-M. Cheng, "Lightweight and flexible graphene foam composites for high-performance electromagnetic interference shielding," Advanced Materials, vol. 25, no. 9, pp. 1296-1300, 2013.

[19] N. J. M. Horing, "Linear graphene plasmons," IEEE Transactions on Nanotechnology, vol. 9, no. 6, pp. 679-681, 2010.

[20] S. A. Mikhailov, "Non-linear electromagnetic response of graphene," EPL (Europhysics Letters), vol. 79, no. 2, p. 27002, 2007.

[21] S. B. Lu, L. L. Miao, Z. N. Guo et al., "Broadband nonlinear optical response in multilayer black phosphorus: An emerging infrared and mid-infrared optical material," Optics Express, vol. 23, no. 9, pp. 11183-11194, 2015.

[22] M. A. Worsley, P. J. Pauzauskie, T. Y. Olson, J. Biener, J. H. Satcher Jr., and T. F. Baumann, "Synthesis of graphene aerogel with high electrical conductivity," Journal of the American Chemical Society, vol. 132, no. 40, pp. 14067-14069, 2010.

[23] Z.-S. Wu, W. C. Ren, L. B. Gao et al., "Synthesis of graphene sheets with high electrical conductivity and good thermal stability by hydrogen arc discharge exfoliation," ACS Nano, vol. 3, no. 2, pp. 411-417, 2009.

[24] X. Gan, K. F. Mak, Y. Gao et al., "Strong enhancement of lightmatter interaction in graphene coupled to a photonic crystal nanocavity," Nano Letters, vol. 12, no. 11, pp. 5626-5631, 2012.

[25] Y. Xiang, X. Dai, J. Guo, S. Wen, and D. Tang, "Tunable optical bistability at the graphene-covered nonlinear interface," Applied Physics Letters, vol. 104, no. 5, Article ID 051108, 2014.
[26] T.-H. Han, Y. Lee, M.-R. Choi et al., "Extremely efficient flexible organic light-emitting diodes with modified graphene anode," Nature Photonics, vol. 6, no. 2, pp. 105-110, 2012.

[27] H. Zhang, D. Tang, R. J. Knize, L. Zhao, Q. Bao, and K. P. Loh, "Graphene mode locked, wavelength-tunable, dissipative soliton fiber laser," Applied Physics Letters, vol. 96, no. 11, Article ID 111112, 2010.

[28] B.-Z. Xu, C.-Q. Gu, Z. Li, and Z.-Y. Niu, "A novel structure for tunable terahertz absorber based on graphene," Optics Express, vol. 21, no. 20, pp. 23803-23811, 2013.

[29] X.-H. Deng, J.-T. Liu, J. Yuan, T.-B. Wang, and N.-H. Liu, "Tunable THz absorption in graphene-based heterostructures," Optics Express, vol. 22, no. 24, pp. 30177-30183, 2014.

[30] L. Jiang, J. Guo, Q. Wang, X. Dai, and Y. Xiang, "Perfect Terahertz Absorption with Graphene Surface Plasmons in the Modified Otto Configuration," Plasmonics, vol. 12, no. 6, pp. 18251831, 2017.

[31] R. Czerw, M. Terrones, J.-C. Charlier et al., "Identification of electron donor states in N-doped carbon nanotubes," Nano Letters, vol. 1, no. 9, pp. 457-460, 2001.

[32] T. Van Khai, H. G. Na, D. S. Kwak et al., "Significant enhancement of blue emission and electrical conductivity of N-doped graphene," Journal of Materials Chemistry, vol. 22, no. 34, pp. 17992-18003, 2012.

[33] Z. Chen, P. Han, C. W. Leung, Y. Wang, M. Hu, and Y. Chen, "Study of optical Tamm states based on the phase properties of one-dimensional photonic crystals," Optics Express, vol. 20, no. 19, pp. 21618-21626, 2012.

[34] H. Zhou, G. Yang, K. Wang, H. Long, and P. Lu, "Multiple optical Tamm states at a metal-dielectric mirror interface," Optics Letters, vol. 35, no. 24, pp. 4112-4114, 2010.

[35] G. W. Hanson, "Quasi-transverse electromagnetic modes supported by a graphene parallel-plate waveguide," Journal of Applied Physics, vol. 104, no. 8, Article ID 084314, 2008.

[36] T. Zhan, X. Shi, Y. Dai, X. Liu, and J. Zi, “Transfer matrix method for optics in graphene layers," Journal of Physics: Condensed Matter, vol. 25, no. 21, Article ID 215301, 2013.

[37] M. Kaliteevski, I. Iorsh, S. Brand et al., "Tamm plasmonpolaritons: Possible electromagnetic states at the interface of a metal and a dielectric Bragg mirror," Physical Review B: Condensed Matter and Materials Physics, vol. 76, no. 16, Article ID 165415, 2007. 

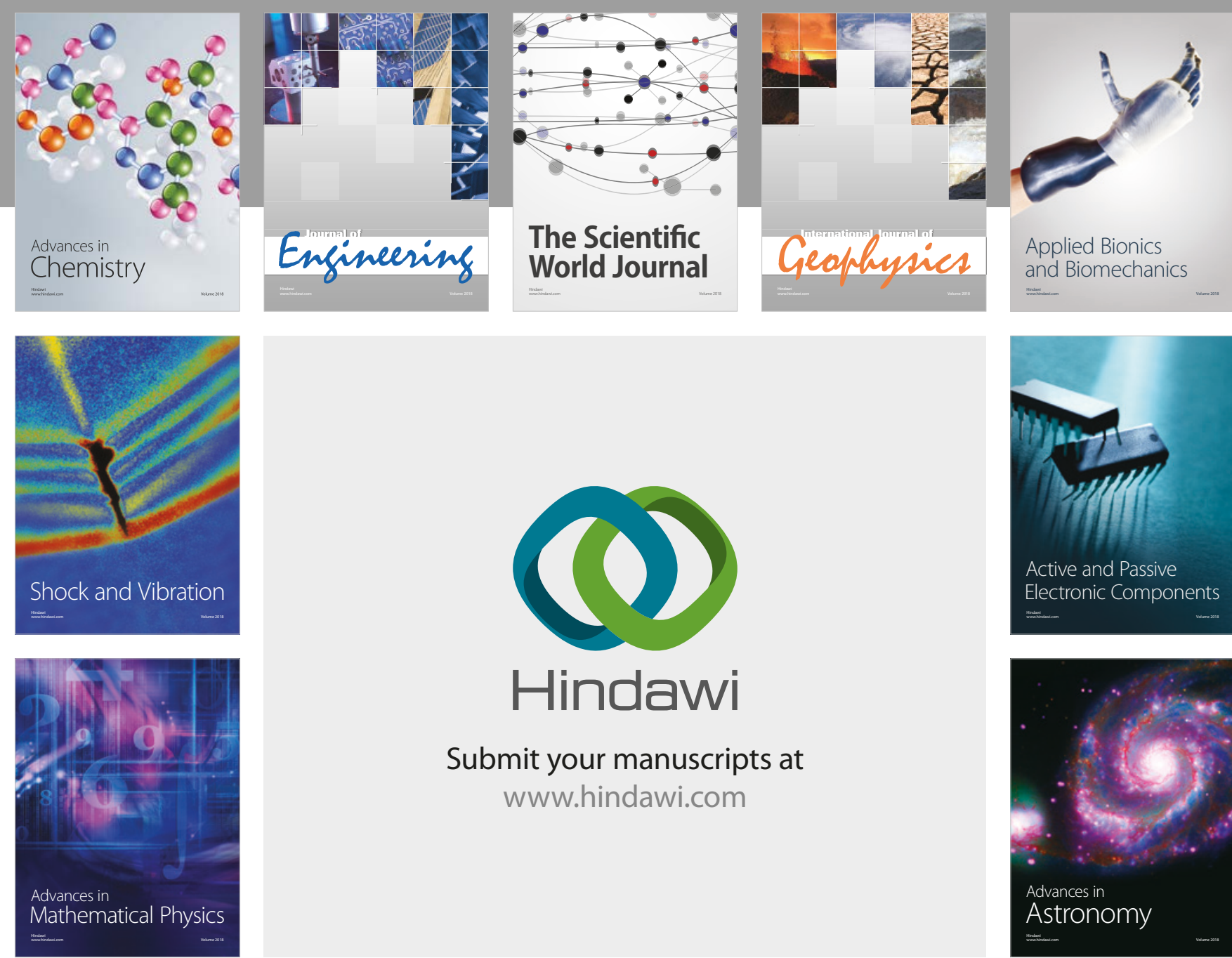

Submit your manuscripts at

www.hindawi.com

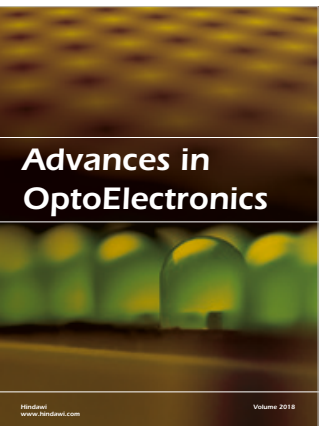

\section{Rotcting Machinery}
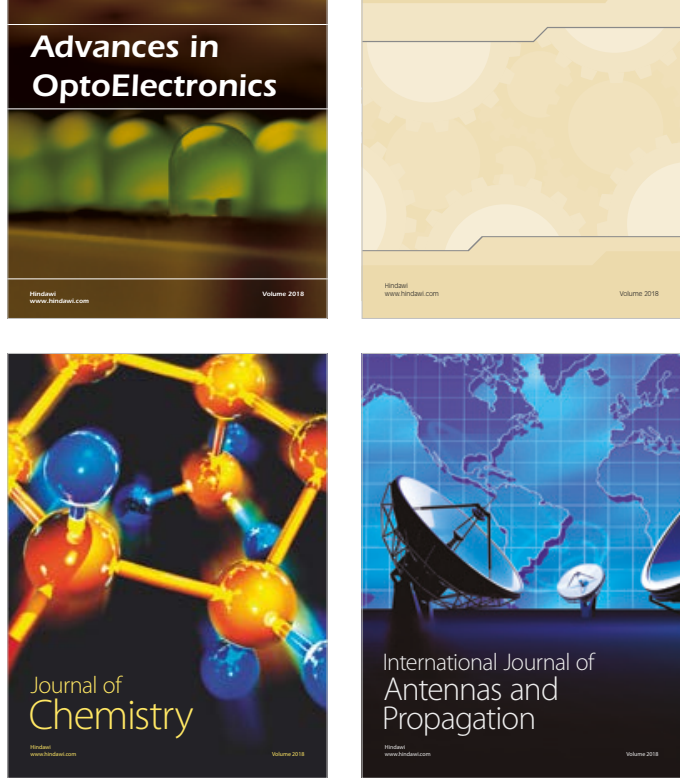

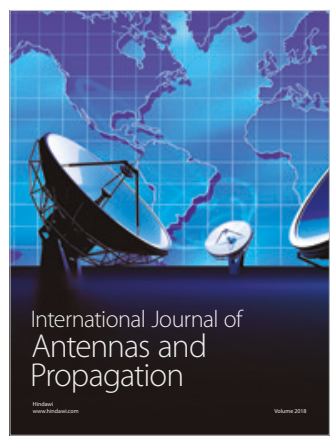

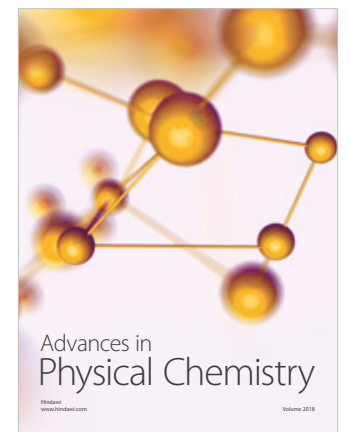

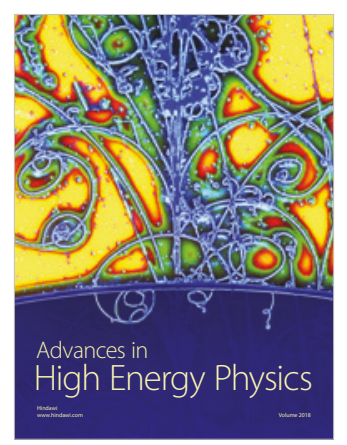

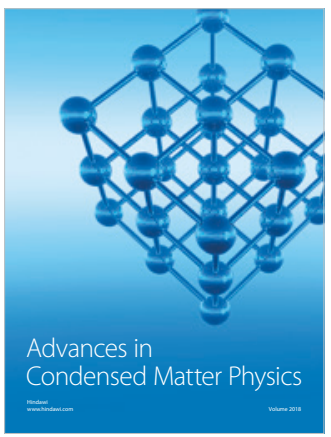

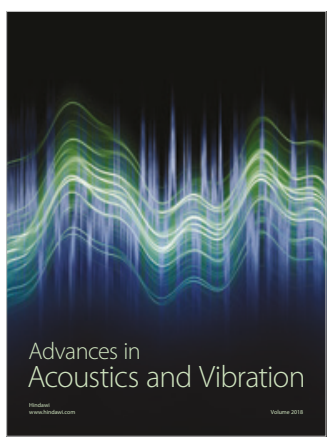

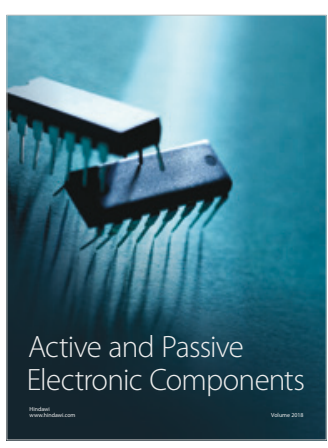
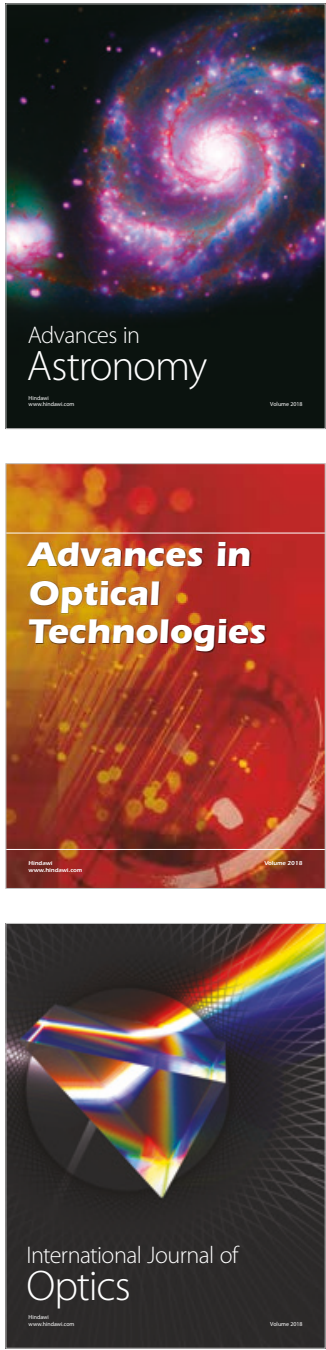